\title{
Les absences au travail en Europe
}

Quel impact du régime d'indemnisation maladie et de la législation de protection de l'emploi sur les comportements des salariés?

Work absences in Europe: what effects of sickness benefits and employment protection legislation on employees' behaviors?

\section{Sabine Chaupain-Guillot et Olivier Guillot}

\section{(2) OpenEdition}

\section{Journals}

Édition électronique

URL : http://journals.openedition.org/travailemploi/1642

DOI : 10.4000/travailemploi. 1642

ISSN : 1775-416X

Éditeur

DARES - Ministère du Travail

Édition imprimée

Date de publication : 15 décembre 2009

Pagination : 17-31

ISSN : 0224-4365

Référence électronique

Sabine Chaupain-Guillot et Olivier Guillot, « Les absences au travail en Europe », Travail et Emploi [En ligne], 120 | octobre-décembre 2009, mis en ligne le 30 décembre 2011, consulté le 01 mai 2019. URL http://journals.openedition.org/travailemploi/1642; DOI : 10.4000/travailemploi.1642 


\title{
Les absences au travail en Europe: quel impact du régime d'indemnisation maladie et de la législation de protection de l'emploi sur les comportements des salariés?
}

\author{
Sabine Chaupain-Guillot(*), Olivier Guillot(*)
}

\begin{abstract}
À partir de données provenant des vagues 3 (1996) et 8 (2001) de l'European Community Household Panel (ECHP), cet article s'intéresse aux déterminants des absences au travail en Europe. L'accent est mis sur les effets de l'indemnisation des arrêts maladie et de la législation en matière de protection de l'emploi. Les absences au cours des quatre dernières semaines, pour raisons de santé ou non, sont analysées à l'aide de régressions logistiques et de type Tobit. D’après les résultats des régressions s'appuyant sur les données de la vague 8 de l'ECHP, le degré de générosité du régime d'indemnisation des arrêts maladie est un élément qui semble jouer positivement sur la probabilité d'absence, aussi bien chez les hommes que chez les femmes. Les résultats obtenus à partir des données de la vague 3 sont, en revanche, plus ambigus. L'étude montre également que le degré de rigueur de la protection de l'emploi a un impact significatif, mais pas dans le sens auquel on pouvait s'attendre. En effet, c'est une relation négative que l'on observe entre l'indice de l'OCDE et la probabilité d'absence.
\end{abstract}

Cette étude s'intéresse aux déterminants des absences au travail dans l'Union européenne à quinze. La question que l'on explore plus particulièrement est celle de l'impact du régime d'indemnisation des arrêts de travail pour maladie et de la législation en matière de protection de l'emploi.

L'absentéisme au travail a fait l'objet de nombreux travaux empiriques (pour une revue, voir Brown, Sessions, 1996). À notre connaissance, une dizaine d'études de comparaison internationale, récentes pour la plupart, ont été menées sur ce thème. Certaines de ces études sont essentiellement descriptives (BergendorfF, 2003; Gimeno et al., 2004; LøKKE, EsKILDSEN, JENSEN, 2007; OsteRKAMP, 2002). D'autres ont cherché à mettre en évidence les déterminants de l'absence au travail, que ce soit à partir de données individuelles (BARMBY, ERCOLANI, Treble, 2002; Bliksvaer, Helliesen, 1997; Frick et MALO, 2005; LeONTARIDI, WARD, 2002) ou au niveau agrégé (Bonato et Lusinyan, 2004; Osterkamp et

(*) BETA (CNRS, université de Strasbourg et université Nancy 2).sabine.chaupain@univ-nancy2.fr ; olivier.guillot@ univ-nancy2.fr. Cet article est issu d'une recherche menée pour le compte de la DARES (Bourreau-Dubors et al., 2008). Les auteurs remercient les deux rapporteurs anonymes, ainsi que les participants aux deux séminaires organisés par la DARES (Paris, 11 décembre 2007 et 15 octobre 2008), pour leurs remarques et suggestions.
RöHN, 2007). Parmi ces travaux, seuls ceux que l'on doit à Bonato et LuSINYAN (2004), Frick et Malo (2005) et OsTERKAMP et RöHN (2007) se sont attachés à estimer les effets de l'indemnisation des arrêts maladie et de la législation en matière de protection de l'emploi sur les absences au travail(1).

Toutes les comparaisons internationales font apparaître des écarts sensibles de taux d'absence entre les pays. Ainsi, au sein de l'Union européenne, d'après les résultats de l'étude menée par GiMENo et al. (2005) à partir des données de l'Enquête européenne sur les conditions de travail de 2000, les taux d'absence pour maladie sont nettement plus élevés en Finlande, en Suède et aux Pays-Bas que dans les pays de l'Europe du Sud. L'impact de l'environnement institutionnel sur les comportements des salariés est l'un des éléments mis en avant par Bonato, Lusinyan (2004) et Osterkamp et RöHN (2007) pour expliquer ces écarts entre pays. Il ressort, en effet, de ces travaux que le degré de générosité du système d'indemnisation des arrêts maladie et le niveau de protection de l'emploi sont positivement liés au taux d'absence (BONATO, LusinYan, 2004) ou au nombre de jours d'absence pour maladie

(1) La question du rôle de l'indemnisation a également été abordée dans des travaux portant sur un seul pays (voir notamment, sur le cas suédois, Henrekson, Persson, 2004, et JohANSSON, PALMe, 2002). 


\section{Encadré 1 \\ La source statistique et le champ de l'étude}

Le Panel européen des ménages (European Community Household Panel (ECHP)) fournit des informations transversales et longitudinales comparables sur les conditions de vie dans les pays de l'Europe des Quinze. Sous la coordination d'EUROSTAT, huit vagues d'enquête ont été réalisées, de 1994 à 2001.

Le questionnaire individuel de ce panel est centré sur le thème de l'emploi. L'une des questions posées aux actifs occupés a porté sur le nombre de jours d'absence au cours des quatre semaines de travail précédant l'enquête. Cette question a été formulée de la manière suivante: "Please think of the last 4 working weeks, not counting holiday weeks. How many days were you absent from work because of illness or other reasons ?". La source utilisée ne permet donc pas de faire la distinction entre les absences pour maladie et celles motivées par d'autres raisons, ni d'isoler le cas des femmes en congé de maternité. En outre, dans la base de données ECHP UDB (Users' Database) mise à disposition par EUROSTAT, le nombre de jours d'absence des actifs travaillant moins de 15 heures par semaine n'est pas connu.

L'analyse proposée ici est une analyse en coupe, menée successivement à partir des données de la vague 3 (1996) de l'ECHP et celles de la vague 8 (2001). La vague 3 est la seule des huit enquêtes annuelles successives à renseigner sur les absences au travail dans l'ensemble des pays de l'Union européenne à quinze, à l'exception de la Suède (1). Pour l'un de ces pays, à savoir l'Autriche, le pourcentage de valeurs manquantes sur le nombre de jours d'absence, dans l'enquête de 1996, est toutefois extrêmement élevé (proche de $50 \%$ ). Ce sont donc treize pays qui ont finalement pu être pris en compte ici. Pour cette étude, on a également jugé intéressant de s'appuyer sur les données les plus récentes de l'ECHP (ie celles de la vague 8), et ce, bien que la comparaison ne puisse dès Iors porter que sur dix des quinze États membres (Belgique, Danemark, Espagne, France, Irlande, Italie, Pays-Bas, Autriche, Portugal et Finlande) (2).

Dans le présent article, le champ a été restreint aux salariés du secteur privé, âgés de 17 à 64 ans (et dont la durée hebdomadaire de travail est d'au moins 15 heures). L'échantillon issu de la vague 3 comprend 29486 individus (18460 hommes et 11026 femmes). Celui provenant de la vague 8, 22778 individus (13799 hommes et 8979 femmes).

(1) Dans le cas de la Suède, cette information n'est disponible pour aucune des huit années.

(2) La vague 8 de l'ECHP n'apporte aucun élément d'information sur les absences au Royaume-Uni et au Luxembourg. Pour l'Allemagne et la Grèce, si le nombre de jours d'absence au cours des quatre dernières semaines est connu, cette information paraît sujette à caution (voir BouRREAu-DuBols et al., 2008, p. 133).

(Osterkamp, RöHn, 2007). Les conclusions de Frick et MALo (2005) vont dans le même sens. Toutefois, ces auteurs soulignent que les variables institutionnelles ont un plus faible impact sur le nombre de jours d'arrêt maladie que certaines caractéristiques individuelles.

L'étude se rapprochant le plus de la présente analyse est celle de FricK et MALO (2005). En effet, comme ces auteurs, c'est à partir de données individuelles que l'on examine les effets de l'indemnisation des arrêts maladie et de la protection de l'emploi sur les comportements d'absence au travail. Frick et Malo, comme Gimeno et al., ont exploité les données de l'Enquête européenne sur les conditions de travail de 2000. La source mobilisée ici est le Panel européen des ménages (ECHP) (voir encadré 1). Ces deux dernières enquêtes, menées l'une et l'autre dans les pays de l'Union européenne à quinze, ne fournissent pas la même mesure de l'absence au travail. En effet, alors que l'Enquête européenne sur les conditions de travail renseigne sur le nombre de jours d'absence pour maladie au cours des douze derniers mois, le questionnaire individuel de l'ECHP permet de savoir si le salarié s'est absenté au cours des quatre dernières semaines, et pendant combien de jours, que cette absence ait été motivée par des raisons de santé ou non. Outre le fait que la présente étude n'est pas centrée sur les absences pour maladie, les deux ensembles de pays considérés ici (comprenant, respectivement, treize et dix États membres de l'Union européenne à quinze) diffèrent quelque peu de celui retenu par Frick et Malo(2).

Dans la première section de cet article, on procède à une brève comparaison des systèmes d'indemnisation des absences pour maladie en vigueur dans les différents pays de l'Union européenne à quinze. Dans la deuxième section, on expose quelques résultats descriptifs, avant d'en venir, dans la troisième section, aux modèles de régression que l'on utilise. Les résultats des estimations sont présentés et commentés dans la quatrième et dernière section.

(2) L'étude de Frick, Malo (2005) porte sur douze pays (Belgique, Danemark, Allemagne, Espagne, France, Irlande, Italie, Pays-Bas, Autriche, Finlande, Suède et Royaume-Uni). 
Tableau 1 : Principales caractéristiques des systèmes d'indemnisation des absences pour maladie - pays de l'Union européenne à quinze (2001)

\begin{tabular}{|l|l|c|l|c|c|}
\hline \multicolumn{1}{|c|}{ Pays } & \multicolumn{1}{|c|}{$\begin{array}{c}\text { Certificat } \\
\text { médical } \\
\text { exigible au } \\
\text { premier jour }\end{array}$} & $\begin{array}{c}\text { Délai de } \\
\text { carence } \\
\text { (en jours) }\end{array}$ & $\begin{array}{c}\text { Maintien intégral } \\
\text { du salaire } \\
\text { par l'employeur }\end{array}$ & \multicolumn{2}{c|}{ Prestations de la protection sociale } \\
\cline { 5 - 6 } & Non & 1 & Oui (7/30 jours) & $60 \%$ du salaire & $\begin{array}{c}\text { Durée maximale } \\
\text { d'indemnisation }\end{array}$ \\
\hline Belgique & Non & 0 & $\begin{array}{l}\text { Selon la convention } \\
\text { collective }\end{array}$ & $\begin{array}{c}\text { Calculé sur la base du } \\
\text { salaire horaire et du nombre } \\
\text { d'heures de travail }\end{array}$ & 52 semaines \\
\hline Danemark & Non & 0 & Oui (6 semaines) & $70 \%$ du salaire & 78 semaines \\
\hline Grèce & Oui & 3 & Non & Forfaitaire & 182,360 ou \\
\hline Espagne & Non & 3 & Non & $60 \%$ du salaire & 12 moirs \\
\hline France & Oui & 3 & $\begin{array}{l}\text { Selon la convention } \\
\text { collective }\end{array}$ & $50 \%$ du salaire & 360 jours \\
\hline Irlande & Oui & 3 & Non & Forfaitaire & 52 semaines \\
\hline Italie & Oui & 3 & $\begin{array}{l}\text { Selon la convention } \\
\text { collective }\end{array}$ & $50 \%$ du salaire & 180 jours \\
\hline Luxembourg & Non & 0 & Oui (3) & $100 \%$ du salaire & 52 semaines \\
\hline Pays-Bas & Oui $(1)$ & 0 & Non & $70 \%$ du salaire & 52 semaines \\
\hline Autriche & Non & 3 & Oui (6 à 12 semaines) & $50 \%$ du salaire & 52 semaines \\
\hline Portugal & Oui & 3 & Non & $65 \%$ du salaire & 1095 jours \\
\hline Finlande & Oui & $0(2)$ & Oui (4) & Jusqu'à $70 \%$ du salaire & 300 jours \\
\hline Suède & Non & 1 & Non & $80 \%$ du salaire & Pas de limite \\
\hline Royaume-Uni & Non & 3 & Non & Forfaitaire & 52 semaines \\
\hline
\end{tabular}

Source: Missoc (2001) (sauf éléments en italique; voir Bourreau-Dubois et al., 2008, p. 130).

(1) Visite de l'inspecteur, possible dès le premier jour.

(2) Le délai de carence de neuf jours s'appliquant aux indemnités de maladie est sans incidence pour le salarié puisque, durant cette période, celui-ci bénéficie du maintien intégral de son salaire.

(3) Pour les employés, maintien intégral du salaire pendant le mois de survenance de la maladie et les trois mois suivants.

(4) Durant le premier jour de maladie et les neuf jours ouvrés suivants.

(5) Dans le cas général.

\section{L'indemnisation des arrêts maladie dans les pays de l'Union européenne à quinze}

La réglementation en matière d'indemnisation des arrêts maladie(3) varie sensiblement selon les pays (voir tableau 1) (4). Quatre éléments retien-

(3) Pour cette mise en parallèle des systèmes d'indemnisation des absences pour maladie, on s'est principalement fondé sur les rapports du Mutual Information System on Social Protection (MISSOC).

(4) L'analyse empirique proposée plus loin s'appuie sur des données qui ont été collectées en 1996 et en 2001. Les dispositifs décrits dans le tableau 1 sont ceux qui étaient en vigueur en 2001. Au cours de la période 1996-2001, à notre connaissance, aucun changement de législation n'est intervenu (excepté en Suède, où le taux d'indemnisation a été porté, en 1998, de 75 à $80 \%$ ). C'est dans la première moitié des années 1990 que d'importantes réformes ont été mises en œuvre aux Pays-Bas et en Suède (voir, sur les Pays-Bas, GeurTs, Kompier, Gründemann, 2000, et, sur la Suède, Henrekson, Persson, 2004). dront ici l'attention: le taux d'indemnisation(5), la durée maximale de versement des indemnités journalières, l'éventuel délai de carence et l'obligation, pour le salarié, de fournir un certificat médical.

En Belgique, en Allemagne, au Luxembourg, en Autriche et en Finlande, le salaire, en cas d'absence pour maladie, est intégralement maintenu par l'employeur. En Espagne, en France, en Italie, aux Pays-Bas, au Portugal et en Suède, le taux d'indemnisation est inférieur ou égal à $80 \%$. Dans deux de ces pays (France et Italie), les indemnités journalières du système de protection sociale peuvent toutefois être complétées par l'employeur, dans les conditions définies par la convention collective (6).

(5) Le taux d'indemnisation peut varier au cours de la période d'arrêt de travail. Il n'est question ici que du taux d'indemnisation applicable au début de l'arrêt maladie, juste après l'éventuel délai de carence.

(6) $\mathrm{Ou}$, dans le cas de la France, par l'accord national interprofessionnel sur la mensualisation. 
Tableau 2: Proportion de salariés ayant été au moins un jour absent, pour des raisons de santé ou non, au cours des quatre dernières semaines (en \%)

\begin{tabular}{|l|c|c|c|c|c|c|}
\hline & \multicolumn{3}{|c|}{$\mathbf{1 9 9 6}$} & \multicolumn{2}{c|}{$\mathbf{2 0 0 1}$} \\
\cline { 2 - 7 } & Hommes & Femmes & Ensemble & Hommes & Femmes & Ensemble \\
\hline Belgique & 12,8 & 16,4 & 14,2 & 13,2 & 17,5 & 15,1 \\
\hline Danemark & 17,5 & 22,8 & 19,3 & 23,9 & 32,0 & 26,7 \\
\hline Allemagne & 11,0 & 9,7 & 10,5 & - & - & - \\
\hline Grèce & 6,1 & 4,4 & 5,5 & - & - & 11,5 \\
\hline Espagne & 9,4 & 10,7 & 9,8 & 9,8 & 14,2 & 9,7 \\
\hline France & 7,9 & 9,9 & 8,7 & 8,6 & 11,3 & 12,6 \\
\hline Irlande & 8,8 & 13,9 & 10,8 & 10,7 & 15,0 & 8,3 \\
\hline Italie & 9,9 & 12,6 & 10,9 & 7,6 & 9,6 & - \\
\hline Luxembourg & 11,5 & 13,5 & 12,3 & - & - & 18,8 \\
\hline Pays-Bas & 18,7 & 22,4 & 19,9 & 16,6 & 22,4 & 8,4 \\
\hline Autriche & - & - & - & 7,8 & 9,3 & 10,0 \\
\hline Portugal & 9,9 & 10,3 & 10,0 & 8,8 & 11,5 & 21,3 \\
\hline Finlande & 17,3 & 23,2 & 19,7 & 19,2 & 24,3 & - \\
\hline Royaume-Uni & 13,1 & 17,9 & 15,1 & - & - & 11,7 \\
\hline Ensemble & 11,1 & 12,9 & 11,8 & 10,4 & 13,7 & \\
\hline
\end{tabular}

Champ: salariés du secteur privé, âgés de 17 à 64 ans, dont la durée hebdomadaire de travail est d'au moins 15 heures.

Source : ECHP UDB - version de décembre 2003, vagues 3 (1996) et 8 (2001), EURSOTAT (calcul des auteurs).

En Irlande, au Royaume-Uni, ainsi qu'en Grèce, c'est un montant forfaitaire qui est versé. Enfin, au Danemark, le montant des indemnités dépend du salaire horaire et du nombre d'heures de travail (le maintien du salaire pouvant être prévu par les conventions collectives)(7).

Limitée à six mois en Italie, la durée d'indemnisation ne peut dépasser une année dans la plupart des autres États de l'Union européenne à quinze. Les trois pays faisant exception sont l'Allemagne (avec une durée maximale de 78 semaines), le Portugal (où la durée d'indemnisation peut atteindre trois ans) et la Suède (où cette durée est illimitée). Au Danemark, en Allemagne, au Luxembourg, aux Pays-Bas et en Finlande, les salariés en arrêt maladie sont indemnisés dès le premier jour d'absence. Dans les autres pays, les indemnités journalières ne sont versées qu'après un certain délai. En Belgique et en Suède, seule la première journée d'absence ne donne pas lieu à compensation. Ailleurs, ce délai de carence est de trois jours.

Dans huit des quinze États membres, aucun certificat médical n'est exigé pour le premier jour d'arrêt maladie. En effet, la production d'un certificat médical n'est requise qu'à partir du deuxième jour en Belgique et au Luxembourg, du quatrième jour au Danemark, en Allemagne, en Espagne et en Autriche, et du huitième jour en Suède et au Royaume-Uni.

(7) Dans ce dernier pays, selon JoHANSEN et al. (2008), les «cols blancs» bénéficient tous du maintien intégral de leur rémunération, tandis que les indemnités perçues par les «cols bleus» sont plafonnées (394 euros par semaine en 2001).
Les systèmes d'indemnisation des absences pour maladie en vigueur en Allemagne et au Luxembourg sont ceux qui présentent, semble-t-il, les niveaux de générosité les plus élevés. Les résultats (non reproduits ici) d'une analyse des correspondances multiples (ACM), portant sur les principales caractéristiques de l'indemnisation, font apparaître un net contraste entre ces deux États et les cinq pays suivants: Grèce, France, Irlande, Italie et Portugal (les systèmes d'indemnisation existant dans ces cinq pays pouvant être considérés comme les moins généreux).

\section{Les absences au travail: éléments descriptifs}

L'analyse des absences au travail est conduite à partir de deux échantillons de salariés du secteur privé, provenant respectivement des vagues 3 (1996) et 8 (2001) de l'ECHP, l'un couvrant treize États membres de l'Union européenne à quinze (les deux pays non retenus étant l'Autriche et la Suède), l'autre, plus restreint, portant sur dix de ces pays (Belgique, Danemark, Espagne, France, Irlande, Italie, Pays-Bas, Autriche, Portugal et Finlande) (voir encadré 1).

En moyenne, dans l'échantillon de 1996 comme dans celui de 2001, ce sont environ $12 \%$ des salariés qui ont été absents au moins un jour, pour raisons de santé ou non, au cours des quatre dernières semaines de travail. Cette proportion est cependant bien plus élevée au Danemark, en Finlande et aux Pays-Bas que dans les autres États membres de l'Union européenne à quinze (hors Suède). En effet, dans ces 
Graphique 1 : Nombre moyen de jours d'absence

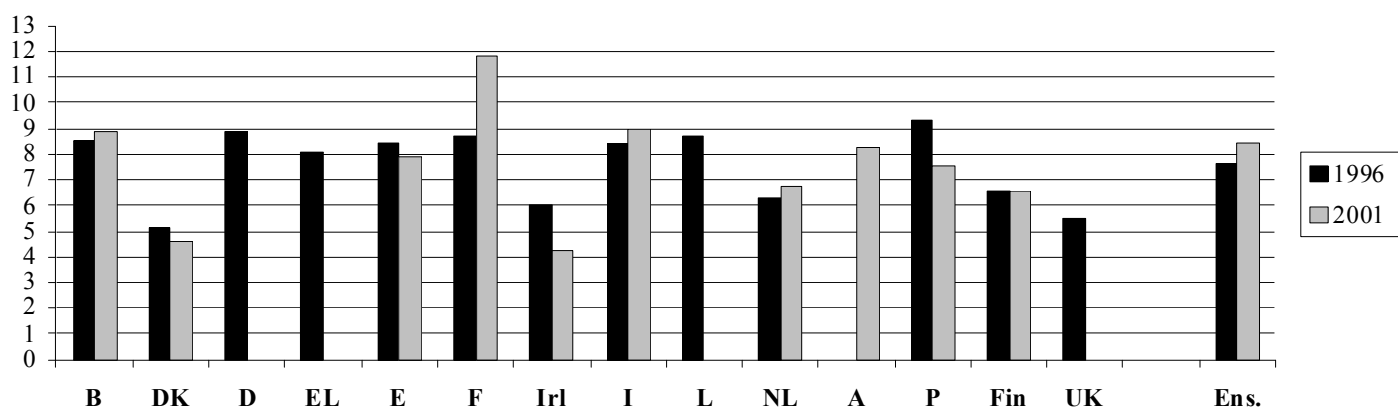

Source: ECHP UDB - version de décembre 2003, vagues 3 (1996) et 8 (2001), EUROSTAT (calculs des auteurs).

Champ: salariés du secteur privé, âgés de 17 à 64 ans, dont la durée hebdomadaire de travail est d'au moins 15 heures, absents au moins un jour au cours des quatre dernières semaines.

Tableau 3 : Taux d'absence selon les caractéristiques de l'emploi occupé (en \%)

\begin{tabular}{|c|c|c|c|c|}
\hline & \multicolumn{2}{|c|}{1996} & \multicolumn{2}{|c|}{2001} \\
\hline & Hommes & Femmes & Hommes & Femmes \\
\hline \multicolumn{5}{|l|}{ Secteur d'activité } \\
\hline Agriculture & 12,5 & (1) & 9,1 & (1) \\
\hline Industrie & 11,2 & 13,5 & 11,2 & 13,3 \\
\hline Tertiaire & 10,6 & 12,4 & 8,8 & 13,4 \\
\hline \multicolumn{5}{|l|}{ Responsabilités d'encadrement } \\
\hline Avec influence sur le salaire/la carrière des subordonnés & 7,7 & 11,1 & 8,8 & 13,9 \\
\hline Sans influence sur le salaire/la carrière des subordonnés & 13,0 & 11,0 & 10,4 & 12,2 \\
\hline Pas de responsabilités d'encadrement & 10,9 & 12,0 & 10,7 & 14,0 \\
\hline \multicolumn{5}{|l|}{ Ancienneté } \\
\hline Moins d'1 an & 9,9 & 9,5 & 11,6 & 10,8 \\
\hline 1 à 5 ans & 12,0 & 13,9 & 10,9 & 15,3 \\
\hline 6 à 10 ans & 10,7 & 15,9 & 10,5 & 16,3 \\
\hline 11 à 15 ans & 9,9 & 11,7 & 9,3 & 14,4 \\
\hline Plus de 15 ans & 11,2 & 10,2 & 9,4 & 11,0 \\
\hline \multicolumn{5}{|l|}{ Type de contrat de travail } \\
\hline CDI & 10,8 & 12,9 & 10,1 & 13,8 \\
\hline CDD & 12,3 & 11,8 & 11,9 & 13,5 \\
\hline \multicolumn{5}{|l|}{ Temps de travail } \\
\hline Temps plein & 11,1 & 12,9 & 10,4 & 13,7 \\
\hline Temps partiel & 13,4 & 12,6 & 9,1 & 14,3 \\
\hline Ensemble & 11,1 & 12,9 & 10,4 & 13,7 \\
\hline
\end{tabular}

Source: ECHP UDB - version de décembre 2003, vagues 3 (1996) et 8 (2001), EUROSTAT (calculs des auteurs).

(1): effectifs insuffisants.

Champ: salariés du secteur privé, âgés de 17 à 64 ans, dont la durée hebdomadaire de travail est d'au moins 15 heures.

trois pays, un salarié sur cinq (plus d'un sur quatre au Danemark, d'après les chiffres de 2001) a été absent (voir tableau 2) (8).

Les femmes sont proportionnellement plus nombreuses à s'être absentées $(12,9 \%$ d'entre elles

(8) Dans le cas du Danemark, on notera que le taux d'absence observé en 2001 est supérieur de 7 points à celui enregistré en 1996 (26,7\% contre 19,3\%). Entre 1994 et 2001, selon une étude du Ministère danois de l'emploi, les dépenses au titre des indemnités de maladie ont augmenté, dans ce pays, de plus de $40 \%$ (BESKeFTIGELSESMINISTERIET, 2003). étant dans ce cas, contre $11,1 \%$ des hommes, dans l'échantillon de $1996 ; 13,7 \%$ contre $10,4 \%$, dans l'échantillon de 2001). À l'échelon des États, on constate que l'écart entre les taux d'absence des hommes et des femmes est significatif dans huit pays en 1996 (Belgique, Danemark, France, Irlande, Italie, Pays-Bas, Finlande et Royaume-Uni) et dans neuf pays en 2001 (Belgique, Danemark, Espagne, France, Irlande, Italie, Pays-Bas, Portugal et Finlande).

Les salariés qui se sont arrêtés, pour raisons de santé ou non, au cours des quatre dernières semaines, 
ont été absents, en moyenne, huit jours(9). La moitié d'entre eux l'ont été, au total, pendant au moins quatre jours. Le nombre moyen de jours d'absence varie selon les pays. En particulier, il est intéressant de noter que dans les états où l'on enregistre les plus forts taux d'absence, à savoir au Danemark, en Finlande et aux Pays-Bas, les salariés ont interrompu leur activité, en moyenne, moins longtemps (voir graphique 1). Par ailleurs, la durée moyenne d'absence est un peu plus élevée chez les femmes que chez les hommes $(8,1$ jours contre 7,2 jours, dans l'échantillon de 1996; 9,2 jours contre 7,8 jours dans l'échantillon de 2001).

Dans l'échantillon de 1996, le taux d'absence des salariés de l'industrie n'est pas significativement différent de celui des salariés du tertiaire. Ce constat vaut pour les hommes comme pour les femmes (voir tableau 3). Les chiffres de 2001 montrent, en revanche, que les hommes qui travaillent dans l'industrie se sont davantage absentés $(11,2 \%$ contre $8,8 \%)$.

Parmi les salariés ayant des responsabilités d'encadrement, une distinction a été opérée dans l'enquête entre ceux qui déclarent exercer une influence sur le salaire ou/et le déroulement de la carrière de leurs subordonnés et ceux qui n'ont pas ce pouvoir. Chez les hommes, on observe que les premiers sont un peu moins nombreux à avoir été absents, pour raisons de santé ou non, au cours des quatre dernières semaines (le taux d'absence de ces salariés étant inférieur à $9 \%$, aussi bien dans l'échantillon de 1996 que dans celui de 2001). Il n'en va pas de même chez les femmes (aucun écart significatif n'étant mis en évidence).

Le taux d'absence des hommes varie peu selon l'ancienneté. Dans le cas des femmes, en revanche, on constate que les absences ont été moins fréquentes parmi les salariées ayant moins d'un an d'ancienneté $(9,5 \%$ contre $13,4 \%$, en moyenne, chez les autres salariées, dans l'échantillon de 1996; 10,8\% contre 14,5\%, dans l'échantillon de 2001). À l'autre extrême, parmi celles qui ont plus de quinze ans d'ancienneté, le taux d'absence est également un peu plus faible.

Les hommes ayant un CDD sont proportionnellement plus nombreux à s'être absentés que les titulaires d'un CDI. Chez les femmes, en revanche, les taux d'absence pour les salariées en CDD et celles en CDI ne sont pas significativement différents. Il n'y a pas non plus d'écart significatif entre le taux d'absence des femmes travaillant à temps partiel (ie effectuant moins de 30 heures par semaine) et celui des actives à temps plein.

(9) La durée moyenne d'absence est de 7,6 jours dans l'échantillon de 1996 et de 8,4 jours dans l'échantillon de 2001.

\section{Les modèles de régression utilisés}

Les comportements d'absence au travail des salariés européens ont été analysés à l'aide de régressions logistiques et de type Tobit (voir encadré 2). La variable dépendante des régressions logistiques prend la valeur 1 lorsque le salarié a été au moins un jour absent, pour raisons de santé ou non, au cours des quatre dernières semaines $(0$ dans le cas contraire). Dans le cadre des modèles Tobit, c'est le nombre de jours d'absence que l'on cherche à expliquer. Les estimations ont été réalisées sur les deux échantillons issus de l'ECHP, et ce, séparément pour les hommes et pour les femmes.

\section{La prise en compte du régime d'indemnisation des arrêts maladie et de la législation sur la protection de l'emploi}

Pour appréhender l'impact éventuel du régime d'indemnisation des arrêts maladie sur les absences au travail, on a introduit - d'abord successivement, puis en les croisant - les trois variables indicatrices suivantes: l'obligation ou non de produire un certificat médical dès le premier jour d'absence, l'existence ou non d'un délai de carence et le fait que le salaire soit ou non intégralement maintenu par l'employeur (voir tableau 1). Une mesure du coût de l'absence pour le salarié (10) et l'indice de générosité proposé par Frick et Malo (voir encadré 3) ont également été successivement utilisés (11). S'agissant de la législation en matière de protection de l'emploi, c'est l'indice synthétique de l'OCDE (1999) (voir encadré 4) qui a été retenu. Cet indice est assez fortement corrélé aux variables ayant trait au système d'indemnisation maladie(12). C'est pourquoi, dans un premier temps, on a estimé des régressions ne prenant en compte que l'indemnisation des absences pour maladie (spécifications 1 à 6) ou, à l'inverse, n'incorporant que l'indice de protection de l'emploi (spécification 7). Le niveau de générosité de l'indemnisation (mesuré, selon le cas, par l'indice de Frick et Malo, à l'aide d'un jeu d'indicatrices croisant les trois critères mentionnés

(10) Seule la perte de salaire (éventuelle) lors du premier jour d'absence a été prise en compte ici. Bien évidemment, lorsque le salaire est intégralement maintenu par l'employeur (et ce, dès le premier jour d'arrêt maladie), le coût de l'absence, ainsi défini, est nul. À l'autre extrême, dans les pays où existe un délai de carence, ce coût est égal au montant total du salaire qui aurait été perçu par l'individu s'il avait travaillé (l'hypothèse d'une journée de travail de 8 heures ayant été retenue).

(11) Dans cette analyse, ce sont les caractéristiques générales du système d'indemnisation qui ont été prises en considération. On ne tient pas compte du fait que, dans certains pays, des conditions plus avantageuses peuvent être prévues par les conventions collectives.

(12) Par exemple, la corrélation (dans les données individuelles, et non pas au niveau pays) entre l'indicateur de l'OCDE et l'indice de Frick et MALO (2005) est de - 0,43 dans l'échantillon de 2001 (cette corrélation étant plus faible et positive $(0,24)$ dans l'échantillon de 1996). 


\section{Encadré 2}

\section{Les régressions de type Logit et Tobit}

Les régressions logistiques sont de la forme:

$$
\log \left[\frac{\operatorname{Pr}\left(Y_{i}=1\right)}{1-\operatorname{Pr}\left(Y_{i}=1\right)}\right]=X_{i} \beta
$$

où $Y_{\mathrm{i}}$ désigne la variable dépendante, codée 1 lorsque l'individu $i$ s'est absenté au moins un jour (0 sinon), $X_{i}$ est le vecteur des variables explicatives et $\beta$, le vecteur des paramètres correspondants (à estimer). La probabilité d'absence est donnée par:

$$
\operatorname{Pr}\left(Y_{i}=1 \mid X_{i}\right)=\frac{\exp \left(X_{i} \beta\right)}{1+\exp \left(X_{i} \beta\right)}
$$

Le recours à des régressions de type Tobit, pour l'analyse de la durée d'absence, vise à tenir compte de la forte proportion de valeurs nulles dans les deux échantillons étudiés.

On pose:

$$
\begin{gathered}
Y_{i}^{*}=X_{i} \beta+\varepsilon_{i} \\
\left\{\begin{array}{l}
Y_{i}=0 \quad \text { si } Y_{i}^{*} \leq 0 \\
Y_{i}=Y_{i}^{*} \text { si } Y_{i}^{*}>0
\end{array}\right.
\end{gathered}
$$

avec $\varepsilon_{i} \sim N\left(0, \sigma^{2}\right)$

où $Y_{i}^{*}$ est une variable latente (non observable), $Y_{i}$ est la variable dépendante observée (ici, le nombre de jours d'absence) et $\varepsilon_{i}$ est un résidu, supposé suivre une loi normale de moyenne nulle et de variance $\sigma^{2}$ ( $X_{i}$ et $\beta$ ont été définis plus haut).

Ces régressions ont été estimées par la méthode du maximum de vraisemblance.

plus haut ou, de manière indirecte et partielle, par le coût de l'absence) et le degré de rigueur de la législation sur la protection de l'emploi ont ensuite été introduits simultanément (spécifications 8 à 10) (13).

\section{Les effets attendus}

On s'attend à ce que le degré de générosité du régime d'indemnisation des arrêts maladie ait un impact positif sur la probabilité d'absence au travail. Le modèle théorique auquel on se réfère ici est celui développé par Allen (1981). Dans ce modèle simple d'arbitrage travail-loisir (modèle qui n'est pas centré sur les arrêts de travail pour

(13) Le système d'indemnisation des arrêts maladie, dans les différents pays européens, s'est sans doute en partie construit sur la base des comportements d'absentéisme observés chez les salariés. Il peut en aller de même pour la législation sur la protection de l'emploi. Dans le cadre de la présente analyse, cette question de l'éventuelle endogénéité des variables institutionnelles n'a pu être explorée. Il convient toutefois de noter que ces variables décrivent des dispositifs qui, en règle générale, ont été mis en place bien avant la date de l'enquête, ce qui réduit, nous semble-t-il, le risque d'endogénéité. maladie, ce qui est cohérent avec l'analyse menée à partir de l'ECHP), l'absence est vue comme un moyen pour les salariés d'ajuster à la baisse leur nombre d'heures de travail, lorsque le temps de travail contractuel est supérieur au nombre d'heures souhaité (ceux-ci étant supposés maximiser leur utilité sous des contraintes de temps et de budget). Les indemnités journalières de maladie, dans le cadre d'un tel modèle, ont pour effet de réduire le coût de l'absence et donc de rendre cet ajustement plus probable.

Parmi les facteurs retenus par Allen figurent également les sanctions éventuelles auxquelles s'exposent les salariés en cas d'absence, un élément jouant négativement (ces sanctions pouvant se traduire par de moins grandes chances de promotion et par un risque accru de licenciement). Cette prédiction du modèle quant au rôle des sanctions conduit, dans le cas présent, à avancer l'hypothèse d'un lien positif entre le degré de rigueur de la législation sur la protection de l'emploi et la propension des salariés à s'absenter.

\section{Les autres variables explicatives}

De nombreuses caractéristiques individuelles ont pu être prises en compte dans cette analyse: l'âge, l'état de santé, l'état matrimonial, le statut d'activité du conjoint éventuel, le nombre d'enfants à charge, l'âge du plus jeune enfant, le fait d'être membre d'une association ou non, le fait de s'occuper ou non (à titre bénévole) d'une personne malade, handicapée ou âgée (14), le revenu du ménage (hors gains de l'individu), le statut d'occupation du logement, ainsi qu'un ensemble de variables ayant trait à l'emploi occupé (le salaire horaire (15), le secteur d'activité, le type de contrat, l'ancienneté, l'exercice éventuel de responsabilités d'encadrement, le fait de travailler ou non à temps partiel et le degré de satisfaction à l'égard des conditions de travail) (16).

(14) Ces quatre dernières variables visant à appréhender l'utilisation du temps «hors travail».

(15) Dans les modèles faisant intervenir la variable de coût (spécifications 5 et 9), le salaire horaire n'a pas été inclus comme régresseur (le coût de l'absence étant fonction du taux de salaire).

(16) On avait initialement ajouté une variable d'environnement, à savoir le taux de chômage au niveau régional (ou national, lorsque l'information sur la région de résidence n'était pas disponible). Bien qu'un effet significatif (négatif) ait pu être observé (surtout chez les hommes), cette variable a finalement été écartée, celle-ci étant par trop corrélée aux variables institutionnelles. Par ailleurs, avant d'introduire les caractéristiques du système d'indemnisation et l'indice de protection de l'emploi, les régressions ont été estimées en incluant, parmi les variables explicatives, un jeu d'indicatrices de pays. Les résultats, non reproduits dans cet article, montrent que la probabilité d'absence des salariés, pour raisons de santé ou non, est plus faible en France que dans la majorité des autres pays européens étudiés. 
Cet indice a été construit à partir des données du MISSOC relatives à l'année 2000. Quatre critères ont été pris en compte: le champ d'application des indemnités journalières de maladie, le délai de carence, la durée maximale d'indemnisation et le taux de remplacement. Chacune de ces variables a été codée de 1 à 3 (du niveau de générosité le plus faible au plus élevé). L'indice repris ici est la moyenne non pondérée de ces quatre variables(1).

\begin{tabular}{|c|c|c|c|c|c|c|c|c|c|c|c|c|c|c|c|}
\hline Pays (2) & 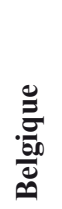 & 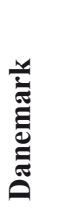 & 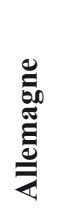 & 芯 & 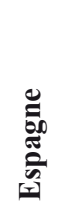 & 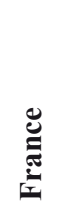 & & 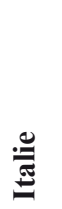 & 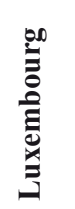 & 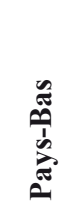 & 葛 & 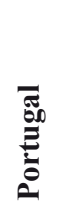 & 冚 & 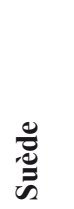 & 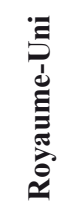 \\
\hline Indice & 2,5 & 2,5 & 2,75 & 1,875 & 2,25 & 2,125 & 1,375 & 1,5 & 2,75 & 2,625 & 2,125 & 2,25 & 2,0 & 2,75 & 1,625 \\
\hline
\end{tabular}

(1) Un second indice a été utilisé par les auteurs: il s'agit d'une moyenne pondérée de ces mêmes variables (les poids retenus étant les suivants: 1 pour le champ d'application et la durée d'indemnisation, 0,5 pour le délai de carence et 1,5 pour le taux de remplacement).

(2) Les valeurs de l'indice pour la Grèce, le Luxembourg et le Portugal ont été calculées par nos soins.

\section{Encadré 4
L'indicateur synthétique de la rigueur de la législation sur la protection de l'emploi (OCDE, 1999)}

Cet indice synthétique, s'appuyant sur la législation en vigueur à la fin des années 1990, a été construit à partir d'une série de 22 indicateurs dits de «premier niveau», convertis en scores variant de 0 à 6 (croissants avec le degré de rigueur de la législation), trois grands domaines étant pris en compte: la réglementation concernant les licenciements individuels de salariés titulaires de contrats permanents (douze indicateurs de premier niveau), les dispositions relatives aux contrats de travail temporaires (six indicateurs) et la réglementation des licenciements collectifs (quatre indicateurs) (pour plus de détails, voir OCDE, 1999, pp. 125-129). La valeur de l'indice est disponible pour vingt-sept pays (dont les États membres de l'Union européenne à quinze, à l'exception du Luxembourg).

\begin{tabular}{|c|c|c|c|c|c|c|c|c|c|c|c|c|c|c|c|}
\hline Pays & ص્ّ & 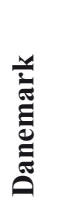 & 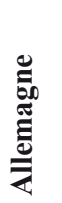 & 芯 & 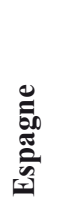 & 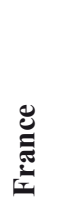 & 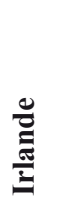 & 駕 & 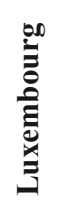 & 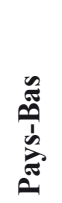 & 苋 & 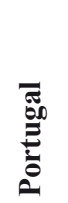 & 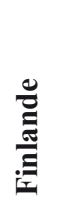 & 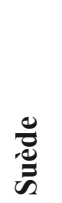 & 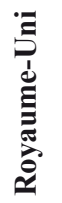 \\
\hline Indice & 2,5 & 1,5 & 2,6 & 3,5 & 3,1 & 2,8 & 1,1 & 3,4 & - & 2,2 & 2,3 & 3,7 & 2,1 & 2,6 & 0,9 \\
\hline
\end{tabular}

\section{Les résultats des estimations}

\section{L'impact des variables d'intérêt}

Si l'on examine tout d'abord les résultats obtenus en introduisant successivement les trois indicatrices ayant trait aux caractéristiques du régime d'indemnisation des arrêts maladie (ie le fait qu'il n'y ait pas d'obligation de produire un certificat médical au premier jour d'arrêt de travail, l'absence de délai de carence et le maintien intégral du salaire par l'employeur - voir tableau 4, spécifications 1 à 3) (17),

(17) Seuls les effets marginaux des variables d'intérêt ont été reportés dans le tableau 4 . Il n'était bien évidemment pas envisageable de fournir ici les résultats complets des nombreuses régressions mises en œuvre (ces résultats étant disponibles auprès des auteurs). À titre d'illustration, on a néanmoins choisi de présenter en annexe, dans le tableau A, les paramètres estimés des régressions logistiques incluant le jeu d'indicatrices relatives au régime d'indemnisation et l'indice de l'OCDE (spécification 8). on constate que, dans les régressions s'appuyant sur l'échantillon de 2001, ces trois variables se sont révélées significatives, aussi bien chez les hommes que chez les femmes, les coefficients estimés étant tous de signe positif. C'est l'effet associé à l'absence de délai de carence qui est le plus marqué. Ainsi, d'après les résultats des régressions logistiques, dans les pays où les indemnités sont versées dès le premier jour de maladie, la probabilité d'absence, pour raisons de santé ou non, est en moyenne de 9 points plus élevée pour les hommes et de 12 points plus élevée pour les femmes, toutes choses égales par ailleurs (18). Lorsque l'analyse est menée

(18) L'effet marginal d'une variable indicatrice $X k$ sur $P$, la probabilité d'absence, a été calculé comme suit: $\Delta P=P\left|X, X_{k}=1-P\right| X, X k=0$ (où $X$ désigne les moyennes des autres variables explicatives). Dans le cas du modèle Tobit, on a (Greene, 2003, pp. 764-768): $\delta E\left[Y_{\mathrm{i}}{ }^{*} \mid X_{\mathrm{i}}\right] / \delta X_{\mathrm{i}}=\beta$. L'effet marginal sur la variable observée est donné par: $\delta E\left[Y_{\mathrm{i}} \mid X_{\mathrm{i}}\right] / \delta X_{\mathrm{i}}=\beta \Phi\left(X_{\mathrm{i}} \beta / \sigma\right)$ (où $\Phi$ est la fonction de répartition de la loi normale). 
à partir de l'échantillon de 1996, on observe un effet de bien moindre ampleur, l'écart de probabilité n'étant que de 2 points chez les hommes et de 1 point (significatif au seuil de $10 \%$ seulement) chez les femmes. S'agissant des deux autres indicatrices, contrairement à ce qui ressort des régressions effectuées sur l'échantillon de 2001, aucun effet significatif n'est mis en évidence pour les hommes. Dans le cas des femmes, si l'indicatrice relative au maintien du salaire apparaît significative, le coefficient de cette variable ne présente pas le signe attendu (l'effet estimé étant négatif, aussi bien dans la régression logistique que dans le modèle Tobit).

En croisant ces trois indicatrices, quatre groupes de pays ont pu être distingués (voir tableau 4, spécification 4) (19). Dans les pays du quatrième groupe (pris comme référence), les salariés sont tenus de produire un certificat médical dès le premier jour d'absence pour maladie, les indemnités journalières sont versées après un délai de carence d'au moins un jour et le salaire n'est pas maintenu par l'employeur (sauf, dans certains pays, si la convention collective le prévoit). À l'opposé, dans les pays du premier groupe, les salariés peuvent s'absenter au moins un jour sans avoir à fournir de certificat médical, il n'y a pas de délai de carence et le salaire est intégralement maintenu. Les systèmes d'indemnisation en vigueur dans les pays formant les groupes 2 et 3 présentent des niveaux de générosité intermédiaires (le degré de générosité étant plus élevé dans le groupe 2 que dans le groupe 3 ).

Dans le cas des hommes, les résultats des estimations montrent que les absences au travail sont plus probables dans le premier groupe de pays que dans le groupe de référence, toutes choses égales par ailleurs. Cet effet ne se retrouve pas chez les femmes, la variable indicatrice relative au groupe 1 étant non significative (dans le modèle Logit comme dans le modèle Tobit)(20). Les indicatrices se rapportant aux groupes 2 et 3 , en revanche, se sont révélées significatives aussi bien chez les hommes que chez les femmes. Dans ces deux groupes de pays, les salariés ont davantage tendance à s'absenter (par rapport au groupe de référence). Les effets marginaux estimés à partir des données de 1996 sont très proches de ceux obtenus en s'appuyant sur les données de 2001. On constate que la probabilité d'absence, pour raisons de santé ou non, est plus forte dans le groupe 2 que dans le groupe

(19) Le premier groupe associe l'Allemagne et le Luxembourg. Le deuxième groupe comprend la Belgique, le Danemark, l'Autriche et la Finlande. Figurent dans le troisième groupe l'Espagne, les Pays-Bas et le Royaume-Uni. Quant au quatrième et dernier groupe, il comprend la France, l'Irlande et les trois autres pays de l'Europe du Sud (Grèce, Italie et Portugal).

(20) Cette indicatrice n'a pu être introduite que dans les régressions s'appuyant sur l'échantillon de 1996 (l'Allemagne et le Luxembourg ne figurant pas parmi les pays étudiés à partir des données de 2001).
3, ce qui correspond à l'effet attendu. Par contre, les salariés des pays du groupe 1 n'ont pas de plus grandes chances d'avoir été au moins un jour absents de leur travail, au cours des quatre dernières semaines, que ceux des pays du groupe 2 , du moins si l'on en juge d'après les résultats des régressions mises en œuvre sur l'échantillon de 1996.

Comme indiqué plus haut, deux autres variables ont été successivement introduites: une mesure du coût de l'absence pour le salarié et l'indice de Frick et Malo. Chez les hommes, la variable de coût est apparue significative aussi bien dans les régressions sur l'échantillon de 1996 que dans celles menées sur l'échantillon de 2001 (voir tableau 4, spécification 5). Dans le cas des femmes, en revanche, seul l'effet mis en évidence à partir des données de 2001 est significatif. Cette variable joue dans le sens attendu: toutes choses égales par ailleurs, plus la perte de salaire lors du premier jour d'arrêt maladie est importante, moins les salariés auraient tendance à s'absenter. D'après les résultats des régressions mises en œuvre sur les données de 2001, l'effet estimé est un peu plus marqué chez les hommes. Ainsi, au point moyen, l'élasticité de la probabilité d'absence par rapport au coût du premier jour d'arrêt de travail est de $-0,25$ pour les hommes, contre $-0,16$ pour les femmes.

Lorsqu'on utilise l'indice de Frick et Malo (voir tableau 4, spécification 6), les résultats que l'on obtient à partir des deux ensembles de données ne sont guère concordants. En effet, l'analyse menée sur l'échantillon de 1996 fait apparaitre un faible effet positif chez les hommes (uniquement dans le modèle Tobit) et, contre toute attente, un effet négatif chez les femmes. Dans l'ensemble des régressions s'appuyant sur les données de 2001, en revanche, l'indice de Frick et Malo est significatif au seuil de $1 \%$. Chez les hommes comme chez les femmes, on observe une relation positive entre le degré de générosité du régime d'indemnisation des arrêts maladie et la propension des salariés à s'absenter, pour raisons de santé ou non, un constat qui rejoint celui formulé par les auteurs, à partir des données de l'Enquête européenne sur les conditions de travail de 2000 (la variable expliquée étant le nombre de jours d'absence pour maladie au cours des douze derniers mois) (21).

Les deux séries de régressions menées ici portent sur des échantillons de pays sensiblement différents, ce qui peut expliquer qu'on aboutisse à des résultats parfois contradictoires. De fait, lorsqu'on restreint le champ de l'analyse aux neuf pays figurant dans les deux ensembles de données (Belgique, Danemark, Espagne, France, Irlande, Italie, Pays-Bas, Portugal

(21) Des régressions avec le second indice proposé par Frick et Malo (voir encadré 3) ont également été estimées. Les résultats vont dans le même sens que ceux présentés dans cet article. 


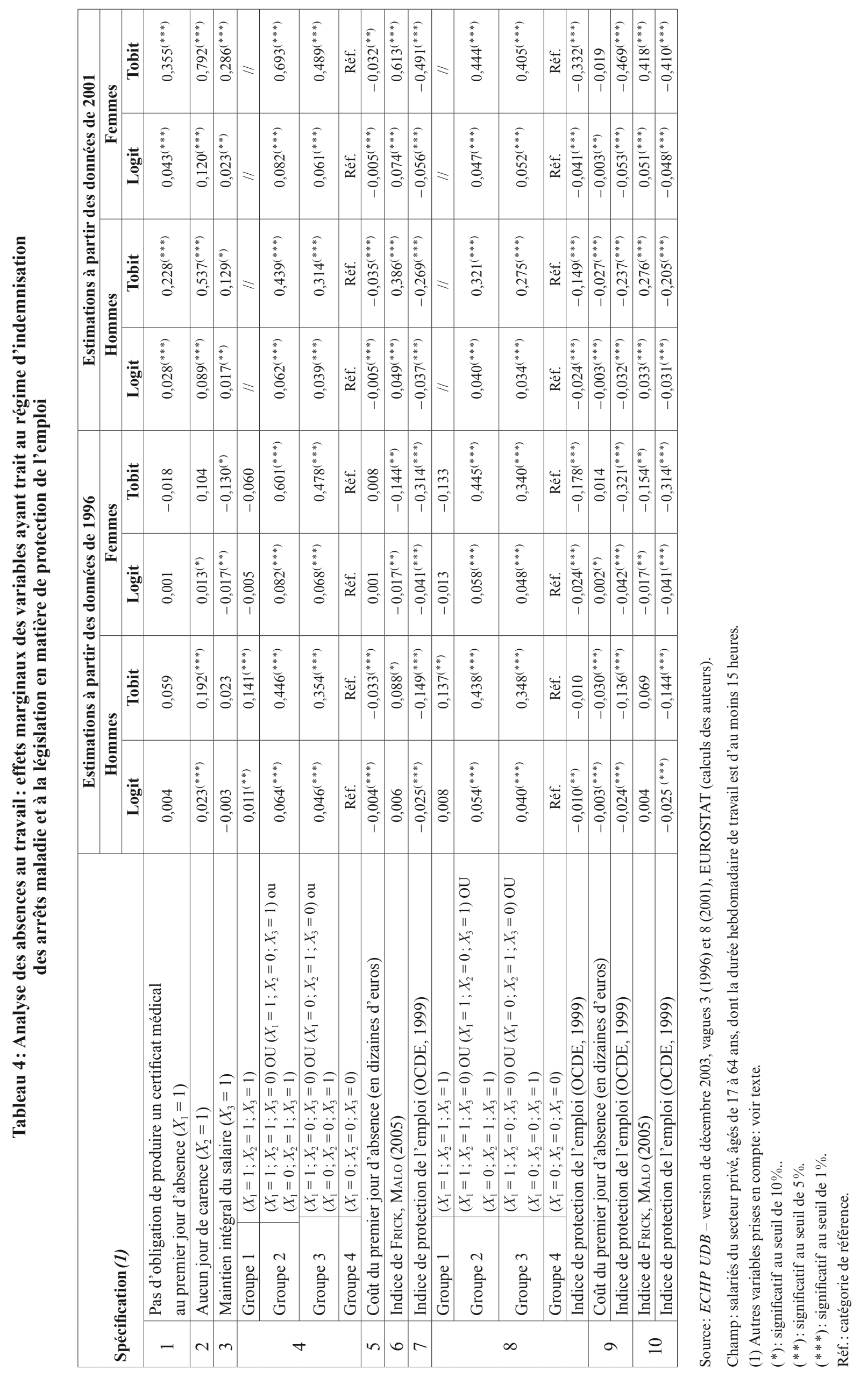

- 26 - Travail et Emploi $n^{\circ} 120$ - Octobre-décembre 2009 
et Finlande), les divergences dans les résultats sont pour l'essentiel gommées. En particulier, les régressions sur les données de 1996 mettent alors en évidence (chez les deux sexes) un lien significatif, et positif, entre l'indice de Frick et Malo et la probabilité d'absence (22).

Si l'on s'intéresse maintenant aux résultats des régressions incluant, comme seule variable institutionnelle, le degré de protection de l'emploi, mesuré par l'indice de l'OCDE (1999) (voir tableau 4, spécification 7), on constate que ce facteur joue négativement (aussi bien en 1996 qu'en 2001, chez les hommes comme chez les femmes, et dans les deux types de modèles)(23). Cet effet négatif, bien que contre-intuitif, n'est guère surprenant si l'on examine les statistiques descriptives. En effet, d'après l'indicateur de l'OCDE (et en se limitant aux pays de l'Union européenne à quinze), c'est dans les états de l'Europe du Sud (Grèce, Espagne, Italie et Portugal) que la législation en matière de protection de l'emploi est la plus rigoureuse. Or, dans ces pays, la proportion de salariés absents au moins un jour au cours des quatre dernières semaines est inférieure à la moyenne européenne. À l'inverse, c'est dans un pays à faible niveau de protection de l'emploi, à savoir au Danemark, que l'on observe le plus fort taux d'absence. L'étude de Frick et Malo conclut également à l'existence d'une relation négative entre le degré de protection de l'emploi et la probabilité d'absence au travail. Toutefois, les résultats de cette étude mettent parallèlement en évidence un effet positif sur le nombre de jours d'absence, effet positif que l'on ne retrouve pas dans les régressions de type Tobit estimées ici. Ce lien négatif entre le degré de rigueur de la législation de protection de l'emploi et la probabilité d'absence des salariés est difficile à interpréter. L'explication que l'on est tenté d'avancer est qu'une législation plus protectrice contribuerait à réduire le stress au travail, ce qui rejaillirait négativement sur les absences (24). Mais les résultats d'une étude menée par WASMER (2006), à partir de données canadiennes, ne corroborent pas cette hypothèse, la protection de l'emploi ayant au contraire, selon cette étude, un impact positif sur le niveau de stress.

Lorsqu'on prend simultanément en compte le degré de générosité du régime d'indemnisation des

(22) Les estimations à partir des données de 1996 ont également été réalisées en excluant successivement certains pays. Chez les hommes, l'indice de Frick et Malo devient significatif dès lors que l'Allemagne n'est plus prise en compte. Pour les femmes, on observe un effet positif (et non plus négatif) si l'on écarte de l'échantillon l'Allemagne et le Royaume-Uni.

(23) Lorsqu'on utilise un autre indicateur, à savoir l'indice du coût des licenciements individuels construit par CADIOU, GUICHARD (1999), on observe un effet de même signe, mais uniquement dans les régressions sur l'échantillon de 2001 (BOURREAU-DUBOIS et al., 2008).

(24) Sur le lien entre stress au travail et absences, voir LeONTARIDI, WARD (2002). arrêts maladie et le niveau de protection de l'emploi (voir tableau 4, spécifications 8, 9 et 10), les conclusions de l'analyse demeurent pour l'essentiel inchangées, les effets marginaux étant cependant, dans la plupart des cas, plus faibles que dans les régressions précédentes.

\section{Le rôle des autres facteurs}

Après s'être intéressé à l'impact des variables institutionnelles, on commente brièvement les résultats relatifs aux autres facteurs (25).

L'état de santé a un rôle déterminant, aussi bien chez les hommes que chez les femmes. Comme attendu, les individus déclarant être gênés dans leurs activités quotidiennes par une maladie chronique ou un handicap ont une probabilité nettement plus élevée que les autres salariés d'avoir été au moins un jour absents au cours des quatre dernières semaines, toutes choses égales par ailleurs. Les femmes ayant au moins un enfant de moins de six ans ont également de plus grandes chances de s'être s'absentées, ce qui traduirait le rôle des contraintes de conciliation entre vie familiale et vie professionnelle (nécessité de rester auprès d'un enfant malade, par exemple) (26). D'après les résultats des régressions s'appuyant sur l'échantillon de 1996, la présence de jeunes enfants joue dans le même sens chez les hommes, l'effet estimé étant cependant de bien moindre ampleur que chez les femmes.

S'agissant de l'impact des caractéristiques professionnelles, on observe que, parmi les salariés en CDI, ceux dont l'emploi a débuté dans l'année ou au cours de l'année précédente sont moins susceptibles de s'absenter, toutes choses égales par ailleurs. Ce résultat, qui vaut surtout pour les femmes, est à rapprocher de celui obtenu par ICHINO et RIPHAHN (2004), les auteurs ayant montré, à partir de données italiennes, que l'absentéisme est significativement plus faible durant la période d'essai. L'analyse menée sur l'échantillon de 1996 révèle que les femmes en CDD ont également moins tendance à s'arrêter (par rapport à la catégorie de référence, ie aux salariées en CDI ayant au moins deux ans d'ancienneté). Chez les hommes, d'après les résultats des estimations s'appuyant sur les données de 2001, le fait d'être en CDD est, au contraire, associé à une probabilité d'absence significativement plus élevée. Dans l'étude de Frick et Malo, c'est un effet négatif qui a été mis en évidence (à partir de régres-

(25) Ce sont les résultats obtenus en introduisant simultanément le jeu d'indicatrices relatives au régime d'indemnisation maladie et l'indice de l'OCDE (1999) qui ont été pris en compte ici.

(26) On notera aussi que certaines de ces femmes ont pu se trouver en congé de maternité au cours des quatre dernières semaines (l'ECHP ne permettant pas de faire la distinction entre de telles absences et les absences pour maladie). 
sions portant sur les salariés des deux sexes) (27). L'exercice de responsabilités d'encadrement, avec droit de regard sur la rémunération ou/et la carrière des subordonnés, a peu d'incidence chez les femmes. Dans le cas des hommes, en revanche, ce facteur joue négativement. L'effet du taux de salaire ne s'est révélé significatif que dans les régressions estimées à partir de l'échantillon de 1996. Chez les hommes, les salariés les mieux rémunérés (ie dont le salaire horaire se situe dans le dernier quartile) ont une plus faible probabilité de s'absenter. Chez les femmes, c'est l'inverse que l'on observe. Enfin, les résultats font apparaître un lien positif entre insatisfaction au travail et absences, et ce, pour les deux sexes.

D'après les résultats des estimations réalisées à partir des données de la vague 8 (2001) de l'ECHP, le degré de générosité du régime d'indemnisation des arrêts maladie est un élément qui semble jouer positivement sur la probabilité d'absence au travail, aussi bien chez les hommes que chez les femmes. Les différentes variables que l'on a successivement introduites pour appréhender cet impact
- simples indicatrices ayant trait aux caractéristiques du système d'indemnisation, jeu d'indicatrices distinguant plusieurs groupes de pays selon le degré de générosité de l'indemnisation, coût d'une journée d'absence pour le salarié, indice de Frick et Malo - se sont toutes révélées significatives, l'effet associé à l'absence de délai de carence étant assez marqué. Les résultats mis en évidence à partir de l'échantillon issu de la vague 3 (1996) sont, en revanche, plus ambigus, plusieurs de ces variables d'indemnisation étant non significatives, voire, contre toute attente, négativement liées à la probabilité d'absence (chez les femmes). Les tests complémentaires qui ont pu être menés montrent que les estimations sont assez sensibles à la composition de l'échantillon de pays.

S'agissant du degré de protection de l'emploi, tel que mesuré par l'indicateur de l'OCDE (1999), l'effet estimé ne va pas dans le sens attendu. En effet, c'est une relation négative qui a été mise en évidence (aussi bien en 1996 qu'en 2001) entre cet indicateur et la probabilité que les salariés aient été au moins un jour absents, un résultat qui rejoint celui obtenu par Frick et Malo. La pertinence d'un tel indicateur peut certes être discutée (KIRAT, 2006). Mais la question du lien théorique entre protection de l'emploi et comportement d'absence demanderait également à être approfondie.

(27) Il convient également de signaler ici que la part des CDD dans l'emploi salarié, lorsqu'elle est introduite comme variable d'environnement, n'a pas d'effet significatif sur les comportements d'absence (au seuil de $5 \%$ ). 


\section{Bibliographie}

Allen S.G. (1981), "An Empirical Model of Work Attendance", Review of Economics and Statistics, Vol. 63, $\mathrm{N}^{\circ} 1$, pp. 77-87.

Barmby T.A., Ercolani M.G., Treble J.G. (2002), “'Sickness Absence: An International Comparison", Economic Journal, Vol. 112, pp. F315-F331.

BergendorfF S. (2003), "Sickness absence in Europe: A comparative study", Paper Presented at the $4^{\text {th }}$ International Research Conference on Social Security, Antwerp, 5-7 May 2003.

BeskÆftigelsesministeriet (2003), “Analyse af det danske sygefravær", København.

Bliksvaer T., Helliesen A. (1997), "Sickness Absence: A Study of 11 LES Countries", LES Working Paper Series, N 3, Luxembourg Employment Study.

Bonato L., Lusinyan L. (2004), "Work Absence in Europe", IMF Working Paper, $N^{\circ}$ 04/193, International Monetary Fund.

Bourreau-Dubois C. et al. (2008), Analyse économique $d u$ droit du travail, rapport final pour le compte de la DARES, BETA, Nancy.

Brown S., Sessions J.G. (1996), "The Economics of Absence: Theory and Evidence", Journal of Economic Surveys, Vol. 10, $\mathrm{N}^{\circ} 1$, pp. 23-53.

Cadiou L., Guichard S. (1999), «La diversité des marchés du travail en Europe: quelles conséquences pour l'Union monétaire?», Document de travail du CEPII, № 1999 10 , Centre d'études prospectives et d'informations internationales.

Frick B., MALO M.A. (2005), “Labour Market Institutions and Individual Absenteeism in the European Union: The Relative Importance of Sickness Benefit Systems and Employment Protection Legislation", mimeo, Faculty of Management and Economics, Witten/Herdecke University.

Geurts S., Kompier M., Gründemann R. (2000), “Curing the Dutch disease? Sickness absence and work disability in the Netherlands", International Social Security Review, Vol. 53, N 4, pp. 79-103.

Gimeno D., Benavides F.G., Benach J., Аmick B.C. (2004), "Distribution of sickness absence in the European Union countries", Occupational and Environmental Medicine, Vol. 61, N 10 , pp. 867-869.

Greene W.H. (2003), Econometric Analysis (5 ${ }^{\text {th }}$ Edition), Prentice Hall, Upper Saddle River.
Henrekson M., Persson M. (2004), “The Effects on Sick Leave of Changes in the Sickness Insurance System", Journal of Labor Economics, Vol. 22, № 1, pp. 87-113.

ICHINO A., RIPHAHN R. (2004), “Absenteeism and employment protection: Three cases studies", Swedish Economic Policy Review, Vol. 11, N 1, pp. 95-114.

Johansen K., Andersen J.S., Mikkelsen S., Pass O., Raffnsøe S., Lynge E. (2008), "Controlling sickness absence: A study of changes in the Danish sickness absence legislation since 1973", Health Policy, Vol. 86, $\mathrm{N}^{\circ} 1$, pp. 109-118.

Johansson P., Palme M. (2002), "Assessing the Effect of Public Policy on Worker Absenteeism", Journal of Human Resources, Vol. 37, $\mathrm{N}^{\circ}$ 2, pp. 381-409.

KIRAT T. (2006), Les indicateurs de protection de l'emploi: la mesure du droit du travail en question?, miméo, Institut de recherche interdisciplinaire en sociologie, économie, science politique, université Paris Dauphine.

LEONTARIDI R.M., WARD M.E. (2002), "Work-related Stress, Quitting Intentions and Absenteeism", IZA Discussion Papers, $\mathrm{N}^{\circ}$ 493, Institute for the Study of Labor.

LøKKe A.-K., EsKildsen J.K., JENSEN T.W. (2007), "Absenteeism in the Nordic countries", Employee Relations, Vol. 29, $\mathrm{N}^{\circ}$ 1, pp. 16-29.

MISSOC (2001), La protection sociale dans les États membres de l'Union européenne et de l'Espace économique européen. Situation au $1^{\text {er }}$ janvier 2001, Commission européenne, Direction générale de l'emploi et des affaires sociales, Office des publications officielles des Communautés européennes, Luxembourg.

OCDE (1999), Perspectives de l'emploi de l'OCDE 1999 - Aider les jeunes à prendre un meilleur départ, OCDE, Paris.

Osterkamp R. (2002), "Work Lost Due to Illness - An International Comparison", CESifo Forum, N ${ }^{\circ} 4 / 2002$, pp. 36-40.

Osterkamp R., RöHn O. (2007), “Being on Sick Leave: Possible Explanations for Differences of Sick-leave Days Across Countries", CESifo Economic Studies, Vol. 53, $\mathrm{N}^{\circ} 1$, pp. 97-114.

Wasmer E. (2006), “The Economics of Prozac: Do Employees Really Gain from Strong Employment Protection?", IZA Discussion Paper, N 2460, Institute for the Study of Labor. 
Tableau A: Analyse des absences au travail : paramètres estimés des régressions logistiques - spécification 8

\begin{tabular}{|c|c|c|c|c|}
\hline & \multicolumn{2}{|c|}{1996} & \multicolumn{2}{|c|}{2001} \\
\hline & Hommes & Femmes & Hommes & Femmes \\
\hline Constante & $-2,331^{(* * *)}$ & $-2,005^{(* * *)}$ & $-2,209^{(* * *)}$ & $-1,424^{(* * *)}$ \\
\hline \multicolumn{5}{|l|}{ Âge } \\
\hline Moins de 40 ans & $0,164^{(* *)}$ & $0,313^{(* * *)}$ & 0,040 & $0,225^{(* *)}$ \\
\hline 40 à 49 ans & Réf. & Réf. & Réf. & Réf. \\
\hline 50 ans ou plus & 0,077 & $-0,244^{(* *)}$ & $-0,158$ & 0,089 \\
\hline \multicolumn{5}{|c|}{ Problème chronique de santé ou handicap/degré de gêne dans les activités quotidiennes } \\
\hline Pas de problème de santé & Réf. & Réf. & Réf. & Réf. \\
\hline Problème de santé-aucune gêne & 0,051 & 0,186 & $-0,049$ & $0,414^{(* *)}$ \\
\hline Problème de santé-gêne légère & $0,793^{(* * *)}$ & $0,977^{(* * *)}$ & $0,926^{(* * *)}$ & $1,033^{(* * *)}$ \\
\hline Problème de santé-gêne sévère & $2,054^{(* * *)}$ & $2,158^{(* * *)}$ & $1,834^{(* * *)}$ & $1,906^{(* *)}$ \\
\hline \multicolumn{5}{|c|}{ Nombre d'enfants (de moins de 18 ans)/âge du plus jeune enfant } \\
\hline Aucun enfant & Réf. & Réf. & Réf. & Réf. \\
\hline 1 enfant - âgé de moins de 6 ans & $0,423^{(* * *)}$ & $0,857^{(* * *)}$ & 0,186 & $0,844^{(* * *)}$ \\
\hline 1 enfant - âgé de 6 ans ou plus & $0,170^{(*)}$ & 0,017 & $-0,097$ & $-0,306^{(* *)}$ \\
\hline 2 enfants - âge du plus jeune $:<6$ ans & $0,256^{(* *)}$ & $0,769^{(* * *)}$ & 0,132 & $0,602^{(* * *)}$ \\
\hline 2 enfants - âge du plus jeune $: \geq 6$ ans & $-0,073$ & $-0,100$ & $-0,107$ & 0,109 \\
\hline 3 enfants ou plus - âge du plus jeune : $<6$ ans & 0,045 & $0,674^{(* * *)}$ & $0,301^{(*)}$ & $1,054^{(* * *)}$ \\
\hline 3 enfants ou plus - âge du plus jeune : $\geq 6$ ans & 0,175 & $-0,244$ & $-0,132$ & $-0,118$ \\
\hline \multicolumn{5}{|l|}{ État matrimonial/statut d'activité du conjoint } \\
\hline Ne vit pas en couple & Réf. & Réf. & Réf. & Réf. \\
\hline En couple - conjoint: actif occupé & $-0,214^{(* * *)}$ & $0,163^{(*)}$ & $0,163^{(*)}$ & $-0,156^{(*)}$ \\
\hline En couple - conjoint: chômeur ou inactif & $-0,078$ & $0,467^{(* *)}$ & $0,308^{(* * *)}$ & $-0,204$ \\
\hline En couple - conjoint: statut d'activité non connu & $-0,084$ & $-0,050$ & $0,551^{(* *)}$ & $-0,293$ \\
\hline \multicolumn{5}{|l|}{ Secteur d'activité } \\
\hline Industrie (ou agriculture) & 0,060 & $0,132^{(*)}$ & $0,224^{(* * *)}$ & 0,047 \\
\hline Tertiaire & Réf. & Réf. & Réf. & Réf. \\
\hline Information manquante & 0,140 & 0,038 & $0,323^{(* * *)}$ & 0,137 \\
\hline \multicolumn{5}{|l|}{ Responsabilités d'encadrement } \\
\hline Avec influence sur le salaire/la carrière des subordonnés & $-0,190^{(* *)}$ & $-0,251$ & $-0,162^{(*)}$ & $-0,032$ \\
\hline Sans influence sur le salaire/la carrière des subordonnés & $0,260^{(* * *)}$ & $-0,151$ & $-0,049$ & $-0,298^{(* * *)}$ \\
\hline Pas de responsabilités d'encadrement & Réf. & Réf. & Réf. & Réf. \\
\hline Information manquante & $-0,211^{(*)}$ & $-0,175$ & $1,053^{(* *)}$ & 0,705 \\
\hline \multicolumn{5}{|l|}{ Type de contrat de travail/année de début de l'emploi } \\
\hline CDI - année de début: avant 2000 & Réf. & Réf. & Réf. & Réf. \\
\hline CDI - année de début: 2000 ou 2001 & $-0,110$ & $-0,427^{(* * *)}$ & 0,081 & $-0,238^{(* *)}$ \\
\hline CDD & $-0,007$ & $-0,199^{(* *)}$ & $0,243^{(* * *)}$ & $-0,060$ \\
\hline Information manquante & $0,447^{(* * *)}$ & $0,743^{(* * *)}$ & $-0,784$ & $-0,887$ \\
\hline \multicolumn{5}{|l|}{ Temps de travail } \\
\hline Temps plein & Réf. & Réf. & Réf. & Réf. \\
\hline Temps partiel & 0,068 & $-0,161^{(* *)}$ & $-0,195$ & $-0,096$ \\
\hline Information manquante & 0,117 & 0,065 & 0,255 & $-0,171$ \\
\hline \multicolumn{5}{|l|}{ Salaire horaire } \\
\hline $1^{\mathrm{er}}$ quartile & Réf. & Réf. & Réf. & Réf. \\
\hline $2^{\mathrm{e}}$ quartile & $-0,102$ & 0,119 & 0,113 & 0,107 \\
\hline $3^{\mathrm{e}}$ quartile & $-0,058$ & 0,040 & $-0,029$ & 0,144 \\
\hline $4^{\mathrm{e}}$ quartile & $-0,303^{(* * *)}$ & $0,370^{(* * *)}$ & $-0,050$ & 0,007 \\
\hline
\end{tabular}




\begin{tabular}{|c|c|c|c|c|}
\hline & \multicolumn{2}{|c|}{1996} & \multicolumn{2}{|c|}{2001} \\
\hline & Hommes & Femmes & Hommes & Femmes \\
\hline \multicolumn{5}{|l|}{ Degré de satisfaction vis-à-vis des conditions de travail } \\
\hline Pas satisfait du tout & $0,526^{(* * *)}$ & $1,051^{(* * *)}$ & $0,550^{(* * *)}$ & $1,717^{(* * *)}$ \\
\hline Pas satisfait & $0,532^{(* * *)}$ & $0,840^{(* * *)}$ & $0,282^{(* *)}$ & $0,790^{(* * *)}$ \\
\hline Pas très satisfait & $0,293^{(* * *)}$ & $0,242^{(* *)}$ & 0,146 & $0,426^{(* * *)}$ \\
\hline Assez satisfait & 0,046 & $0,226^{(*)}$ & 0,171 & 0,150 \\
\hline Satisfait & $-0,007$ & 0,040 & 0,148 & 0,063 \\
\hline Très satisfait & Réf. & Réf. & Réf. & Réf. \\
\hline Information manquante & 0,415 & $-0,054$ & 0,274 & $-0,339$ \\
\hline Appartenance à une association & $0,129^{(* *)}$ & $0,135^{(* *)}$ & $0,160^{(* * *)}$ & $-0,016$ \\
\hline Soins à une personne malade, handicapée ou âgée & $-0,070$ & $-0,409^{(* *)}$ & $\left.0,426^{(*)}\right)$ & $-0,336^{(*)}$ \\
\hline \multicolumn{5}{|c|}{ Revenu mensuel du ménage, hors gains d'activité de l'individu } \\
\hline $1^{\mathrm{er}}$ quartile & Réf. & Réf. & Réf. & Réf. \\
\hline $2^{\mathrm{e}}$ quartile & $0,238^{(* * *)}$ & $-0,180^{(*)}$ & 0,033 & 0,059 \\
\hline $3^{\mathrm{e}}$ quartile & $0,130^{(*)}$ & $-0,251^{(* * *)}$ & 0,050 & $0,187^{(*)}$ \\
\hline $4^{\mathrm{e}}$ quartile & 0,099 & $-0,111$ & 0,030 & $-0,057$ \\
\hline \multicolumn{5}{|l|}{ Statut d'occupation du logement } \\
\hline Propriétaire & $-0,346^{(* * *)}$ & $-0,200^{(* *)}$ & $-0,188^{(* *)}$ & $-0,190^{(* *)}$ \\
\hline Accédant & $-0,151^{(* * *)}$ & $-0,305^{(* * *)}$ & $-0,145^{(*)}$ & $-0,170^{(* *)}$ \\
\hline Locataire (ou sous-locataire) & Réf. & Réf. & Réf. & Réf. \\
\hline Logé à titre gratuit & $-0,129$ & $0,264 *$ & 0,023 & $-0,166$ \\
\hline \multicolumn{5}{|l|}{ Régime d'indemnisation des arrêts maladie } \\
\hline Groupe 1 & 0,104 & $-0,155$ & // & $/ /$ \\
\hline Groupe 2 & $0,561^{(* * *)}$ & $0,538^{(* * *)}$ & $0,460^{(* * *)}$ & $0,447^{(* *)}$ \\
\hline Groupe 3 & $0,437^{(* * *)}$ & $0,462^{(* * *)}$ & $0,395^{(* * *)}$ & $0,485^{(* * *)}$ \\
\hline Groupe 4 & Réf. & Réf. & Réf. & Réf. \\
\hline Indice de l'OCDE (1999) & $-0,111^{(* *)}$ & $-0,251^{(* * *)}$ & $-0,280^{(* * *)}$ & $-0,397^{(* * *)}$ \\
\hline Logarithme de la vraisemblance & $-5999,23$ & $-3797,18$ & $-4363,15$ & $-3268,05$ \\
\hline
\end{tabular}

Source: ECHP UDB - version de décembre 2003, vague 3 (1996) et vague 8 (2001), EUROSTAT (calculs des auteurs).

Champ: salariés du secteur privé (âgés de 17 à 64 ans).

${ }^{(*)}$ : significatif au seuil de $10 \%$.

${ }^{(*)}$ : significatif au seuil de $5 \%$.

${ }^{(* *)}$ : significatif au seuil de $1 \%$.

Réf. : catégorie de référence. 